7th International Workshop on Astronomy and

Relativistic Astrophysics (IWARA 2016)

International Journal of Modern Physics: Conference Series

Vol. 45 (2017) 1760014 (4 pages)

(C) The Author(s)

DOI: $10.1142 / \mathrm{S} 201019451760014 \mathrm{X}$

\title{
The Cosmic Censorship and Kantowski-Sachs Model
}

\author{
Patrícia Carvalho, Miguel Campos \\ Departamento de Física, Universidade Federal de Roraima (UFRR) \\ Campus do Paricarana, RR, Brazil \\ patriciacarvalho.fisica@gmail.com, miguel.campos@ufrr.br \\ Brisa Terezón \\ Departamento de Física, Universidade Federal de Roraima (UFRR) \\ Campus do Paricarana, RR, Brazil \\ Departamento de Ciencias Básicas, Universidad Don Bosco \\ Calle Las Claveles, San Salvador, El Salvador \\ bterezon@gmail.com
}

Published 15 August 2017

Let's discuss some points, concerning to the cosmic censorship conjecture for the Kantowski-Sachs model.

Keywords: Kantowski-Sachs Model, Cosmic Censorship Conjecture, Collapse Process.

PACS numbers: 97.60.-s, 95.35.+d, 97.60.Lf, 98.80.C

\section{Introduction}

Let us deal briefly here with two important themes that can be correlated: The spherically symmetric and anisotropic model of Kantowski-Sachs, and the cosmic censorship conjecture. The Kantowski-Sachs model is interesting because of its topology somewhat different from those usually associated with models that populate the Bianchi classification, i.e., the model is resulting from the product of a sphere with a line $\left(R^{3} \times R\right)$. It can be describe by as a spatially homogeneous and anisotropic model that does not permit a simple transitive group of motions. Cosmological expanding solutions to these models are known and can be found in the original paper of Kantowski-Sachs ${ }^{1}$ and in Kramer's book, ${ }^{2}$ with the matter content represented by a perfect fluid, where all solutions have singularities.

On the other hand, it has been shown that considering a positive cosmological constant in Kantowski-Sachs universes, the model admit inflationary solutions, in

This is an Open Access article published by World Scientific Publishing Company. It is distributed under the terms of the Creative Commons Attribution 4.0 (CC-BY) License. Further distribution of this work is permitted, provided the original work is properly cited. 
which we have an isotropization process, i.e., shear decays exponentially towards zero. It is also possible to find in the literature, inhomogeneous solutions with inflation directed by a cosmological constant. Hence, in general, the universe can start with a very inhomogeneous and anisotropic phase, then go us through the inflationary era, subjecting homogenization and isotropization.

We can classify spacetime singularities according to whether or not can be observed. If the singularity can be observed it is called a naked singularity, while a black hole is a typical example of a spacetime singularity that cannot be observed. Related with this question, Roger Penrose proposed the cosmic censorship conjecture that have two versions. The weak conjecture state that all singularities in gravitational collapse are hidden within a black hole. On the other hand, the strong conjecture asserts that no singularity visible to any observer can exist.

\section{Kantowski-Sachs Collapse}

Usually the Kantowski-Sacks metric is put in the form

$$
d s^{2}=d t^{2}-A^{2}(t) d r^{2}-B^{2}(t)\left[d \theta^{2}+\sin ^{2} \theta d \phi^{2}\right] .
$$

We consider the light velocity equal to unity and the energy momentum tensor as a perfect fluid:

$$
T^{\mu \nu}=(\rho+p) u^{\mu} u^{\nu}-p g^{\mu \nu},
$$

where $\epsilon$ is the energy density, $p$ is the pressure and $u$ is the four-velocity of the star fluid.

Let us consider the case of dust, which allows us to write the field equations as:

$$
\begin{gathered}
2 \frac{\ddot{B}}{B}+\frac{\dot{B}^{2}}{B^{2}}+\frac{1}{B^{2}}=0, \\
\frac{\ddot{A}}{A}+\frac{\ddot{B}}{B}+\frac{\dot{A}}{A} \frac{\dot{B}}{B}=0, \\
2 \frac{\dot{A}}{A} \frac{\dot{B}}{B}+\frac{\dot{B}^{2}}{B^{2}}+\frac{1}{B^{2}}=8 \pi G \rho .
\end{gathered}
$$

The first integral of Eq.(3) results

$$
\dot{B}^{2}=b\left(\frac{B_{i}}{B}\right)-1,
$$

where $b=1+\left(B_{i} H_{B i}\right)^{2}, H_{B i}=\frac{\dot{B}}{B}(t=0)$ and $B_{i}=B(t=0)$.

To obtain the full integration of the Eq.(3) we introduce the auxiliary variable

$$
u=\frac{1}{b}\left(\frac{B}{B_{i}}\right) \text {, }
$$

in Eq.(6), resulting

$$
\dot{u}=\frac{1}{b B_{i}}(1-u / u)^{1 / 2}
$$


Making the reversal of above equation, we have

$$
\frac{d t}{d u}=b B_{i}\left(\frac{u}{1-u}\right)^{1 / 2}
$$

that associate to the second derivative, results in

$$
u(1-u) \frac{d^{2} t}{d u^{2}}-\frac{1}{2} \frac{d t}{d u}=0 .
$$

This equation is a particular case of the hypergeometric differential equation with parameters $\left(0,-1,-\frac{1}{2}\right)$, whose solution is given by

$$
t(u)=c_{1}+c_{2} u^{3 / 2} F(1 / 2,3 / 2,5 / 2 ; u),
$$

where $c_{1}$ and $c_{2}$ are integration constants.

Considering the initial conditions

$$
\begin{gathered}
B(t=0)=B_{i}, \\
B=0 \quad \text { at } t=t_{c},
\end{gathered}
$$

we rewrite Eq.(11) as

$$
1-\frac{t}{t_{c}}=\left(\frac{B}{B_{i}}\right)^{3 / 2} \frac{F(1 / 2,3 / 2,5 / 2 ; B / b B)}{F(1 / 2,3 / 2,5 / 2 ; 1 / b)},
$$

where the collapsing time can be calculated from Eq.(12):

$$
t_{c}=\frac{H_{B_{i}}^{-1}\{2 b F(1 / 2,3 / 2,5 / 2 ; 1 / b)\}}{3 b F(1 / 2,3 / 2,5 / 2 ; 1 / b)+\frac{3}{5} F(3 / 2,5 / 2,7 / 2 ; 1 / b)},
$$

and where, in addition, we also use the initial condition

$$
t=0 \quad \Rightarrow \quad H_{B}=-H_{B_{i}} .
$$

Alternatively, we can use the contiguous relation

$$
\frac{d}{d Z}\left\{Z^{a} F(a, b, c, z)\right\}=\frac{\Gamma(a+1)}{\Gamma(a)} Z^{a-1} F(1+a, b, c ; z),
$$

in Eq.(12), to find a more simple expression for the collapsing time, namely

$$
t_{c}=\frac{2 F(1 / 2,3 / 2,5 / 2 ; 1 / b)}{3 H_{B_{i}} F(1 / 2,5 / 2,5 / 2 ; 1 / b)} .
$$

We can reduce this expression for the collapsing time in terms of usual functions

$$
t_{c}=\frac{1}{H_{B_{i}}}\left\{1-b+b(b-1)^{1 / 2} \sin ^{-1}\left(b^{-1 / 2}\right)\right\} .
$$

This collapses process, in some sense, mimics the collapse of a fluid with positive curvature, because the initial value for the Hubble function can be set to zero:

$$
H_{B_{i}} \rightarrow 0 \Rightarrow t_{c}=\pi / 2 \text {. }
$$

In Fig. 1 we display the evolution of the scalefactor $B$. 


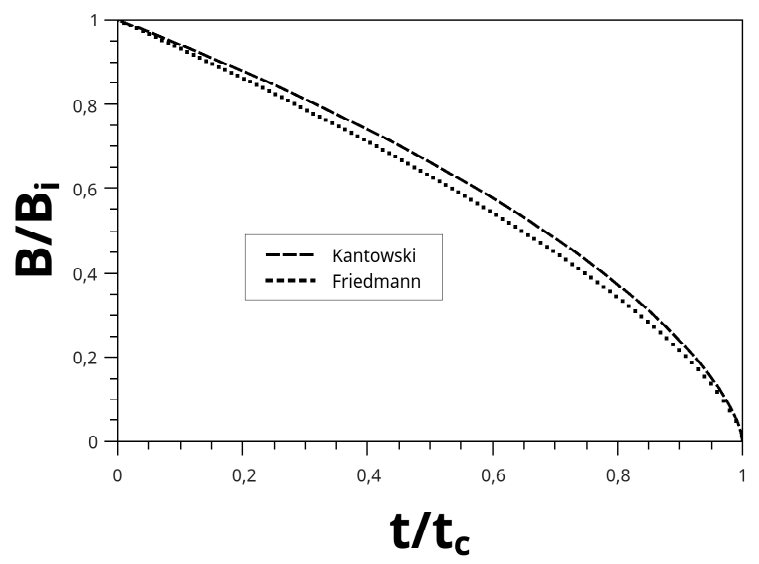

Fig. 1. Evolution of the scalefactor $B$ versus adimensional time, where we compare the collapse process for the scalefactor $\mathrm{B}(\mathrm{t})$ (Kantowski-Sachs scalefactor) with the evolution for the scalefactor taking into account the usual FRW model, where $\frac{R}{R_{0}}=\left(1-t / t_{c}\right)^{2 / 3}$.

\section{Conclusions and Some Notes}

Our intent in this work is study the collapse process taking into account a Kantowski-Sachs spacetime. Under determined initial conditions the scalefactor $B$ collapses, as we can view in the Fig.(1). In addition to the scalefactor B, we also have $\mathrm{A}$, and in this case we intend to make the approximation via the Kretschmann scalar, that for the metric given by Eq.(1) results

$$
K=\left(\frac{\ddot{A}}{A}\right)^{2}+2\left(\frac{\ddot{B}}{B}\right)^{2}+\left(2 \frac{\dot{A} \dot{B}}{A B}\right)^{2}+\frac{1}{B^{4}}+2\left(\frac{\dot{B}}{B^{2}}\right)^{2}+\frac{\dot{B}^{4}}{B} .
$$

Another point that will merit our attention as an extension of this work is to consider a charged fluid, and in a second step include a vacuum term. Both will be useful as a counterexample of the cosmic censorship conjecture; and in relation to the collapsed mass, we can have increase or decrease of this quantity, depending on both what is included in the stellar fluid as well as the definition of mass used. These are, approximately, the next steps that we will take for the study of anisotropic collapse.

\section{References}

1. R. Kantowski and R. K. Sachs, J. Math. Phys. 7, 443 (1966).

2. D. Kramer, H. Stephani, M. MacCallum, E. Hertt, Exact Solutions of Einstein's Field Equations (Cambridge University Press, New York, 1980). 